Revue internationale P.M.E.

\title{
Les stratégies tête-de-pont : un outil efficace de gestion des risques et d'accélération du développement international des
}

PME?

\section{Gateway strategies : an efficient tool to manage risks and accelerate SMEs' internationalization? \\ Estrategias cabeza-de-ponte : un instrumento de gestion de riesgo y de internationalizacion para las PyME ?}

\section{Noémie Dominguez}

Volume 29, numéro 3-4, 2016

URI : https://id.erudit.org/iderudit/1038334ar

DOI : https://doi.org/10.7202/1038334ar

Aller au sommaire du numéro

Éditeur(s)

Editions EMS - In Quarto SARL

ISSN

0776-5436 (imprimé)

1918-9699 (numérique)

Découvrir la revue

Citer cet article

Dominguez, N. (2016). Les stratégies tête-de-pont : un outil efficace de gestion des risques et d'accélération du développement international des PME ? Revue internationale P.M.E., 29(3-4), 109-134. https://doi.org/10.7202/1038334ar
Résumé de l'article

La saturation des marchés traditionnels et l'ouverture des économies poussent les PME à se développer sur les marchés étrangers. Bien que souffrant d'un manque réel de ressources, la part grandissante des PME optant pour la réalisation d'un investissement direct à l'étranger (IDE) appelle à une relecture des stratégies d'internationalisation de ces petites structures. Cet article repose sur l'analyse de cinq cas de PME manufacturières de la région Rhône-Alpes ayant ouvert une filiale à l'étranger. Il présente les principaux risques supportés par les PME et les motivations au déploiement d'une stratégie tête-de-pont. Le choix d'une implantation dans un pays tête-de-pont s'avère être une démarche défensive ou offensive résultant de l'interaction de facteurs endogènes et exogènes. Nos résultats montrent que les stratégies tête-de-pont constituent une stratégie efficace permettant à la fois de gérer les risques et de saisir de nouvelles opportunités d'affaires à l'étranger - notamment dans les pays émergents. 


\title{
Les stratégies tête-de-pont : un outil efficace de gestion des risques et d'accélération du développement international des PME?
}

\begin{abstract}
Noémie DOMINGUEZ
Noémie Dominguez est maître de conférences en sciences de gestion à l'IAE Lyon - Université Jean Moulin Lyon 3. Elle enseigne le management international et est responsable du Master 1 International Business Realities. Dans la lignée de sa thèse, soutenue en 2015, elle s'intéresse aux nouvelles stratégies d'internationalisation des PME et, plus précisément, aux stratégies tête-de-pont.
\end{abstract}

Centre de recherche Magellan IAE Lyon, Université Jean Moulin Lyon 3

6, cours Albert Thomas

69008 LYON, France noemie.dominguez@univ-lyon3.fr

\begin{abstract}
RÉSUMÉ
La saturation des marchés traditionnels et l'ouverture des économies poussent les PME à se développer sur les marchés étrangers. Bien que souffrant d'un manque réel de ressources, la part grandissante des PME optant pour la réalisation d'un investissement direct à l'étranger (IDE) appelle à une relecture des stratégies d'internationalisation de ces petites structures. Cet article repose sur l'analyse de cinq cas de PME manufacturières de la région Rhône-Alpes ayant ouvert une filiale à l'étranger. Il présente les principaux risques supportés par les PME et les motivations au déploiement d'une stratégie tête-depont. Le choix d'une implantation dans un pays tête-de-pont s'avère être une démarche défensive ou offensive résultant de l'interaction de facteurs endogènes et exogènes. Nos résultats montrent que les stratégies tête-de-pont constituent une stratégie efficace permettant à la fois de gérer les risques et de saisir de nouvelles opportunités d'affaires à l'étranger - notamment dans les pays émergents.
\end{abstract}

MOTS-CLÉS

Internationalisation, Risques, PME, Stratégies tête-de-pont

\section{Gateway strategies : an efficient tool to manage risks and accelerate SMEs' internationalization?}

\begin{abstract}
The saturation of traditional markets and the opening-up of economies push SMEs to internationalize. Even if they suffer from resources scarcity, more and more SMEs are investing abroad, calling for a reinvestigation of their expansion strategies. This article relies on the analysis of five independent manufacturing SMEs having (at least) one subsidiary abroad. It aims at understanding the main motivations pushing SMEs to implement gateway strategies in order to penetrate foreign markets. The decision to invest in a gateway country is following a defensive or offensive logic resulting from the interaction of external and internal factors. It appears that gateway strategies act as a tool to facilitate the entry to emerging market, reducing at the same time SMEs' exposure to local risks.
\end{abstract}

KEYWORDS

Internationalization, Risks, SMEs, Gateway strategies 


\title{
Estrategias cabeza-de-ponte : un instrumento de gestion de riesgo $y$ de internationalizacion para las PyME?
}

\begin{abstract}
RESUMEN
La saturación de los mercados tradicionales y la apertura de las economías empujan PyME a internacionalizar. Si suffren de una falta real de recursos, la parte creciente de las PyME que opta por Inversión en el extranjero llama a una segunda lectura de las estrategias de internacionalización de estas estructuras. Este artículo repone en el análisis de cinco casos de PyME de la región francesa Rhône-Alpes que han abierto una filial en el extranjero. Presenta los principales motivos que explican el recurso a una estrategia indirecto de penetracion de los mercados, llamadas estrategia cabeza-de-ponte. La elección de un establecimiento en un país cabeza-de-pont se revela ser un paso defensivo u ofensivo resultando de la interacción de factores endógenos y exógenos. Nuestros resultados muestran que las estrategias cabeza-de-pont constituyen un instrumento que facilita el acceso a los mercados emergentes reduciendo, al mismo tiempo, la exposición de la PyME a los riesgos locales.
\end{abstract}

Palabras Clave

Internacionalización, Peligros, PyME, Estrategias cabeza-de-ponte

\section{INTRODUCTION}

Spécialiste français de l'éclairage LED, la petite entreprise Lucibel a créé l'événement en annonçant, le 29 avril 2014, sa décision d'ouvrir une filiale à Casablanca (Lucibel Africa) pour pénétrer rapidement les marchés africains. Les enjeux liés à l'accès à l'énergie en Afrique et les plans d'investissements gouvernementaux et supranationaux engagés en ce sens témoignent du fort potentiel de la zone. Cette implantation stratégique offre à Lucibel, selon les dirigeants, "l'opportunité d'accroître son développement sur un marché favorable aux énergies renouvelables et à la transition énergétique. Par la qualité de ses infrastructures routières et portuaires, le Maroc est une plateforme stratégique pour adresser l'Afrique de l'Ouest et le Maghreb. Son implantation au Royaume du Maroc permettra à Lucibel d'améliorer la qualité du service offert à ses clients, d'augmenter sa présence commerciale sur le terrain et d'accroître sa visibilité. La présence de Lucibel au Maroc ne restera pas uniquement commerciale. Nous souhaitons rapidement transmettre notre connaissance et notre savoir-faire dans l'éclairage LED. Le Royaume du Maroc, avec sa volonté et sa vocation d'ouverture sur les pays africains, est une excellente opportunité de développement de Lucibel sur l'ensemble du continent africain » (Frédéric Granotier, président-directeur général de Lucibel, 29 avril 2014).

Le fort potentiel de développement des pays africains a ainsi poussé Lucibel à définir de nouvelles stratégies d'approche en vue de gagner rapidement des parts de marché localement. Souvent écartés pour leur relative instabilité, les pays d'Afrique constituent en effet des cibles de plus en plus prisées par les entreprises occidentales dans la mesure où l'intensité concurrentielle y est faible, mais les besoins importants. La question se pose alors de savoir comment concilier deux concepts a priori opposés, à savoir poursuivre l'expansion internationale et cibler des pays à fort potentiel de croissance tout en limitant, dans le même temps, l'exposition aux risques locaux. L'exemple du cas de la PME Lucibel montre qu'aujourd'hui, 
le choix de la localisation n'apparaît plus seulement dicté par le potentiel de croissance du pays, mais bien par celui de l'ensemble des marchés environnants, renvoyant ainsi au concept de stratégie tête-de-pont. Selon Javalgi, Deligonul, Ghosh, Lambert et Cavusgil (2010) la sélection de la localisation et le niveau d'engagement de l'entreprise "ne sont pas seulement influencés par le potentiel de croissance du [marché cible], mais aussi par sa capacité à servir de porte d'accès vers d'autres marchés [voisins] » (Javalgi et al., 2010, p. 209).

Bien que souvent analysée dans le cadre des firmes multinationales, l'internationalisation des activités concerne également les petites et moyennes entreprises (PME), celle-ci tendant aujourd'hui à devenir leur axe central de développement (Lemaire, 2013). L'élargissement des marchés et l'importance croissante des pays émergents offrent de nouvelles opportunités de développement que de plus en plus de PME choisissent de saisir (St-Pierre, Defays, Benezech et Garcia, 2014). Caractérisées par leurs ressources limitées et une certaine vulnérabilité face à l'environnement (Lu et Beamish, 2001), les PME se voient en effet contraintes de s'internationaliser par la taille restreinte du marché domestique, l'intensification de la concurrence ou encore le besoin d'accéder à de nouvelles ressources et compétences (Lemaire, 2013 ; Bertrand et St-Pierre, 2015).

Si le développement international des PME a souvent été étudié sous le prisme de l'export, il s'avère toutefois qu'elles sont de plus en plus nombreuses à opter pour des modes d'entrée plus engageants tels que la coentreprise, l'acquisition d'entités étrangères ou la création de filiales en vue de s'implanter durablement sur les marchés étrangers. Les statistiques de l'INSEE (2015) montrent qu'en 2014, près de $11 \%$ des filiales étrangères d'entreprises françaises sont détenues par des PME indépendantes. Ceci témoigne de la complexification grandissante des stratégies d'internationalisation des PME face à l'évolution de l'environnement au sein duquel elles opèrent. L'internationalisation ne doit donc plus être uniquement considérée comme étant mue par la conquête de nouveaux marchés ou de nouvelles parts de marché, mais bien comme une stratégie visant à répartir efficacement la chaîne de production et d'approvisionnement des PME entre différents espaces économiques pour tirer le meilleur parti des facteurs qu'ils offrent et desservir la clientèle locale de la façon la plus efficace et la moins coûteuse (Lemaire, 2013). Comme l'indiquent Leyronas et Torrès (1996), il semble ainsi que l'internationalisation tend à dénaturer les PME, celles-ci adoptant des comportements atypiques aux logiques semblables à celles des grandes entreprises. Les stratégies dites «tête-de-pont » s'inscrivent dans cette lignée. Ces nouvelles stratégies proactives d'expansion - offensive ou défensive, dont le niveau d'engagement est guidé par la volonté de l'entreprise de prendre pied sur ce marché afin de pénétrer des marchés proches plus vastes, remettent en question la validité des modèles théoriques existants.

Prenant appui sur l'exemple de Lucibel au Maroc, l'objectif de cet article est de montrer en quoi les stratégies tête-de-pont constituent, aujourd'hui, un mode de gestion efficace des risques à l'international. Si les théories classiques de l'internationalisation des PME mettent essentiellement l'accent sur le rôle de l'expérience, l'aversion au risque, ou encore le manque de ressources et compétences pour expliquer les trajectoires d'expansion de ces entreprises, il n'existe pas à notre connaissance de travaux portant sur l'efficacité des stratégies tête-depont comme solutions palliatives des risques internationaux. Cette étude, basée sur l'analyse de cinq cas de PME manufacturières de la région Rhône-Alpes ayant opté pour une approche indirecte des marchés, nous a permis de conclure que la mise en œuvre d'une stratégie têtede-pont pouvait être tant proactive que réactive, répondant à des problématiques d'origine 
interne comme externe. Cette approche s'avère tout d'abord préférée par les PME souhaitant pallier la perte de compétitivité causée par les mesures protectionnistes locales ainsi que celles souhaitant tirer profit de l'image positive du pays d'implantation dans le pays cible. En d'autres termes, elle permet aux PME de réduire leur exposition aux risques locaux tout en préservant leur accès aux marchés cibles. Le déploiement des stratégies tête-de-pont semble, par ailleurs, influencé par les caractéristiques des produits, l'expérience internationale, les relations du réseau ou encore la vision du dirigeant.

Dans une première partie, nous analyserons les fondements théoriques relatifs aux stratégies d'internationalisation des entreprises, en focalisant notre attention sur les éléments explicatifs des trajectoires d'expansion ainsi que sur le choix de la localisation. Dans une deuxième partie, la justification du cadre méthodologique sera suivie par une présentation des PME manufacturières retenues pour mener à bien cette étude de cas. La troisième partie sera consacrée à la comparaison des résultats obtenus lors de l'étude. Nous conclurons en présentant les principaux apports, limites et perspectives de cette recherche.

\section{REVUE DE LITTÉRATURE}

\subsection{L'approche processuelle}

L'internationalisation des PME est un phénomène multidimensionnel pouvant être appréhendé par le biais de différents cadres théoriques. Demeurant l'une des principales approches mobilisées depuis les travaux de Cyert et March (1963), l'approche processuelle renvoie à une suite logique de choix incrémentaux concernant la sélection des marchés cibles et des modes d'entrée. Le modèle d'Uppsala présente ainsi l'avantage d'être, encore aujourd'hui, l'un des seuls outils permettant d'analyser simultanément le processus de sélection des marchés et des modes d'entrée (Meier et Meschi, 2010). Dans sa forme initiale, le modèle d'Uppsala explique le caractère progressif de l'expansion des firmes sur les marchés étrangers en s'appuyant sur les notions de connaissances expérientielles et d'engagement. L’engagement graduel répond au besoin de minimiser le fort degré d'incertitude perçu par les firmes dû à l'absence de connaissances relatives aux marchés étrangers, en phase initiale d'expansion (Johanson et Wiedersheim-Paul, 1975 ; Johanson et Vahlne, 1977). L'apprentissage constitue une dimension clé dans la réussite de l'entreprise à l'étranger - notamment dans les marchés émergents - celle-ci tendant à débuter son internationalisation en pénétrant les marchés présentant la plus faible distance psychique ${ }^{1}$ (Angué et Mayrhofer, 2010). La réplication des schémas d'expansions fructueux aux différents marchés cibles entraîne alors une augmentation séquentielle des ressources allouées à l'international et du degré d'engagement local.

1 Johanson et Vahlne (1977, p. 24) définissent la distance psychique comme étant «l'ensemble des facteurs bloquant le flux d'informations entrants et sortants du marché. Ceux-ci comprennent les différences de langage, de formation, de pratiques managériales, de culture et de développement industriel ». Cette définition sera complétée en 2009, les auteurs incluant la religion au concept de distance psychique. 
L'harmonisation grandissante des marchés et le développement des nouvelles technologies de communication contribuent à diminuer les effets de la distance sur les acteurs ainsi que la prégnance de l'expansion par le marché. Dans ses deux nouvelles versions, le modèle d'Uppsala $(2009,2013)$ stipule que l'internationalisation de l'entreprise est la conséquence d'un ensemble de décisions stratégiques, essentiellement mue par la volonté d'intégrer de nouveaux réseaux d'affaires ${ }^{2}$. La question centrale réside désormais dans l'identification du réseau à intégrer, celui-ci favorisant la réussite de l'expansion et diminuant les effets négatifs du manque de connaissances et de la distance psychique (Métais, Véry et Hourquet, 2010). Bien que présentant certains risques - tels que le manque de transparence dans les relations, l'opportunisme ou encore la dépendance vis-à-vis de certains membres clés, etc. - il constitue une source importante d'informations fiables et pertinentes, permettant à l'entreprise d'identifier et saisir de nouvelles opportunités ainsi que développer des relations de confiance à long terme avec des partenaires étrangers. La non-appartenance au réseau est alors perçue comme un handicap pour la firme souhaitant se développer à l'international, faisant écho à ce que Johanson et Vahlne (2009) qualifient de "liability of outsidership ». L'accumulation d'expérience résultant des connexions établies avec les membres identifiés comme ayant une position centrale dans le réseau étranger renforce le capital confiance de l'entreprise, favorisant alors le développement de nouvelles connaissances (Coviello et Munro, 1997 ; Nahapiet et Ghoshal, 1998 ; Johanson et Vahlne, 2009 ; Vahlne et Johanson, 2013).

Les récents travaux de Figueira-de-Lemos, Johanson et Vahlne (2011) apportent un éclairage nouveau sur la relation risque-engagement dans le processus d'internationalisation. Dans la lignée des travaux existants en entrepreneuriat international, les auteurs affirment que l'entreprise désireuse de se développer à l'étranger accorde désormais davantage d'importance à la maximisation des profits qu'à la minimisation de l'incertitude. Lorsqu'elle œuvre en environnement stable et que le niveau de risque supporté s'avère inférieur au maximum toléré, l'entreprise tend à se surexposer volontairement au risque en augmentant son niveau d'engagement - jusqu'à l'atteinte de son seuil de tolérance - afin de maximiser ses profits sur le marché. La confiance joue un rôle clé dans la décision d'engagement en ce qu'elle permet d'atténuer les effets de l'incertitude comportementale, facilitant ainsi le passage d'une étape à l'autre. Casson et Da Silva Lopes (2013) apportent un éclairage spécifique quant aux différents types de risques auxquels les PME sont exposées. Selon ces auteurs, les entreprises sont confrontées à trois types de risques de nature subjective, institutionnelle et naturelle (Tableau 1).

2 Le réseau d'affaires est ici défini comme étant «l'ensemble des relations variées, proches et durables de la firme avec des fournisseurs et des clients importants " (Johanson et Vahlne, 2009, p. 1414). 
TAbleau 1. Ty Pologie de Risques RenContrés PAR Les PME À L'Étranger

\begin{tabular}{|c|c|c|}
\hline Type de risque & Classification & Description \\
\hline Subjectif & Méconnaissance & $\begin{array}{l}\text { Accidents causés par la sous-évaluation des facteurs } \\
\text { naturels, des changements inattendus de réglementations et } \\
\text { mesures restrictives, sous-estimation des coûts, etc. }\end{array}$ \\
\hline \multirow[t]{4}{*}{ Institutionnel } & Politique & $\begin{array}{l}\text { Instabilité ou modification brutale et inattendue de la sphère } \\
\text { politique, guerre, expropriation, taxation punitive, non- } \\
\text { reconnaissance ou violation de la propriété intellectuelle par } \\
\text { les gouvernements locaux, etc. }\end{array}$ \\
\hline & Social & $\begin{array}{l}\text { Troubles à l'ordre public (sabotages, violence, grève...), } \\
\text { modification des attentes sociales, boycottage, etc. }\end{array}$ \\
\hline & Affaires & $\begin{array}{l}\text { Qualité et disponibilité des fournisseurs locaux, de la } \\
\text { main-d'œuvre, des infrastructures et des services publics ; } \\
\text { existence de substituts, manque de complémentarité } \\
\text { des produits ou services, apprentissage local, imitation, } \\
\text { violation des brevets et des marques, monopole des réseaux } \\
\text { de distribution, matières premières, etc. }\end{array}$ \\
\hline & Financier & $\begin{array}{l}\text { Pertes de capital dues aux faillites, fraudes, volatilité des } \\
\text { marchés financiers, hyperinflation, volatilité des taux de } \\
\text { change, dépréciations monétaires, taux d'intérêt locaux, } \\
\text { modification du niveau général des prix, etc. }\end{array}$ \\
\hline Naturel & Climatique & $\begin{array}{l}\text { Tempêtes, inondations, tremblements de terre, famines, } \\
\text { épidémies, etc. }\end{array}$ \\
\hline
\end{tabular}

Source : adapté de Casson et Da Silva Lopes (2013).

Bien que très répandu dans la littérature en management international, le modèle d’Uppsala a fait l'objet de vives critiques au fil des ans, nombre de chercheurs lui reprochant son aspect déterministe faisant obstacle à l'existence de tout processus alternatif (Coviello et McAulley, 1999 ; Forsgren, 2002), le caractère difficilement mesurable des indicateurs utilisés (Evans et Mavondo, 2002) ou encore l'absence d'intégration des typologies d'entreprise et des spécificités sectorielles (Andersen, 1993 ; Ojala et Tyrväinen, 2007). Face à l'émergence de comportements atypiques, Oviatt et McDougall (1994) remettent en cause le caractère incrémental du processus et l'impact de la distance psychique en affirmant que certaines firmes - baptisées born global - se développent de façon rapide et multimarchés dès leur création. L'existence d'une forte expérience antérieure à l'international du dirigeant ou de l'organisation, la démocratisation des technologies d'information et de communication (TIC), l'harmonisation des marchés ou encore la flexibilité des jeunes entreprises sont autant de facteurs expliquant l'apparition de ce type de comportement (Pantin, 2010).

L'obtention d'un avantage concurrentiel significatif par l'exploitation des ressources détenues en interne et la création de valeur sont les deux principaux moteurs de l'expansion multipays de ces structures (Oviatt et McDougall, 1994). Le choix de la localisation dépend dans ce cas du potentiel du pays à valoriser les produits de l'entreprise. En d'autres termes, s'il est principalement dicté par des besoins de minimisation de l'incertitude et d'accès au 
réseau dans le cas du modèle d'Uppsala, le choix de la localisation semble davantage lié à la recherche de maximisation de la valeur dans le cas des born global (Meier et Meschi, 2010). Le concept d'entreprises born-again global, proposé par Bell, McNaughton et Young (2001), vient enrichir le débat entre internationalisation rapide et incrémentale. Les auteurs stipulent que l'internationalisation rapide ne concerne pas uniquement les jeunes entreprises : certaines entreprises traditionnelles tendent elles aussi à se développer de façon rapide à l'international après une longue période de concentration sur leur marché domestique. Ce changement soudain s'explique par l'acquisition de ressources uniques (tangibles et/ou intangibles), l'accès à un nouveau réseau à l'étranger, ou tout autre incident critique tel que le rachat de l'entreprise (Bell, McNaughton, Young et Crick, 2003 ; Jean-Amans, 2011).

Outre la vitesse et la trajectoire d'expansion, les entreprises born et born-again global se différencient de leurs homologues traditionnelles sur le plan des risques auxquels elles sont exposées. Dans un effort de conceptualisation de l'internationalisation rapide des PME, Weerawardena, Mort, Liesch et Knight (2007) ont mis en lumière le rôle clé des partenaires et des réseaux. Selon eux, la sélection du partenaire constitue un risque majeur pour ces entreprises dans la mesure où, au regard de la spécificité de leur mode de croissance, elles se voient contraintes de recourir à une grande diversité d'intermédiaires locaux. Les réseaux constituent alors un élément efficace en vue de pallier ce risque et d'accéder aux informations, aux ressources et aux compétences requises pour réussir une internationalisation rapide. Les travaux conduits par Gabrielsson, Kirpalani, Dimitratos, Solberg et Zucchella (2008) vont plus loin en mettant en avant le fait que les entreprises à internationalisation rapide font face à des risques différents et plus complexes que ceux supportés par les PME traditionnelles. Les entreprises traditionnelles sont principalement exposées à un type de risque : le risque lié au marché cible. Les born-global doivent, pour leur part, relever un double défi : le lancement simultané de nouveaux produits sur de nouveaux marchés, tout en tenant compte des contraintes liées au manque de ressources disponibles et au caractère vital de la croissance rapide pour ces entreprises. L'interaction de ces différents éléments crée des tensions nouvelles et encore peu étudiées dans la littérature (Gabrielsson et al., 2008).

\subsection{Les stratégies tête-de-pont et IDE plateformes}

Les profondes mutations de la sphère économique ont généré l'apparition de nouveaux comportements à l'international et poussent les chercheurs à reconsidérer les critères de sélection de la localisation (Javalgi et al., 2010 ; Nguyen, 2011). Les accords de coopération noués entre les différents pays participent en effet à la globalisation des échanges et sont vecteurs de nouvelles opportunités d'affaires. Selon Ekholm, Forslid et Markusen (2007), les coûts de fragmentation - coûts supplémentaires engendrés par le fait de produire à l'étranger plutôt qu'à domicile - et l'appartenance à une zone d'intégration économique constituent les principaux déterminants dans le choix d'une approche indirecte des marchés. Les auteurs affirment ainsi que l'entreprise tend à recourir à une stratégie tête-de-pont dans deux cas de figure distincts : lorsque le pays d'accueil présente des avantages significatifs en termes de coûts - de production, de transport, de transaction et des coûts de fragmentation modérés - ou lorsqu'il permet à l'entreprise extérieure à la zone de coopération de s'en rapprocher. La localisation dans un pays voisin permet de réduire les coûts de transport et de 
production tout en se rapprochant de la demande, sans avoir à supporter les risques liés à un IDE dans une zone instable. Dans cette même lignée, Javalgi et al. (2010) s'interrogent sur l'impact des accords de libre-échange sur le processus d'internationalisation, en prenant l'exemple des FMN nord-américaines investissant au Mexique. Selon eux, la décision d'IDE au Mexique est influencée par les possibilités d'accès aux marchés émergents d'Amérique latine. L'intégration dans une zone de libre-échange et l'amélioration de l'environnement local - privatisations, disparition progressive des barrières tarifaires et des mesures restrictives, gain de stabilité économique, etc. - font du Mexique un pays tête-de-pont intéressant pour accéder à des marchés tiers fluctuants et distants du pays d'origine. Cette approche facilite l'accès aux marchés d'Amérique latine porteurs et réduit les effets négatifs du risque et de la distance globale sur le décideur, notamment grâce à la mise à profit des connaissances expérientielles développées par l'entreprise et/ou ses dirigeants dans le pays tête-de-pont.

Nguyen (2011) propose une typologie des principales motivations, analysées sous un angle macroéconomique, justifiant la concrétisation d'un IDE plateforme ${ }^{3}$. L’adoption d'une approche indirecte résulte de la combinaison de cinq facteurs : le marché (potentiel de croissance et accessibilité), le travail (ressources humaines qualifiées à bas coût), la stabilité politique et les politiques locales en matière d'IDE, et les autres facteurs (concurrence, qualité des fournisseurs, accès aux ressources naturelles). Le faible degré de développement des infrastructures locales et le coût élevé des services de base tendent à inhiber la décision d'investir dans le pays cible au profit du pays tête-de-pont (Minda et Nguyen, 2012).

Si les approches théoriques classiques tendent à expliquer le rythme d'expansion et le choix de la localisation des PME par le besoin de minimisation de l'incertitude ou la maximisation de la valeur, elles s'avèrent insuffisantes pour expliquer la mise en œuvre des stratégies tête-de-pont et dans quelle mesure ces stratégies constituent un mode de gestion efficace des risques à l'international. Dans la même lignée, si la littérature relative aux IDE plateformes offre un éclairage intéressant quant à l'influence des conditions environnementales, elle ne tient cependant pas compte des facteurs endogènes ayant un impact sur le choix de la stratégie d'expansion. Dès lors, la combinaison de ces deux approches complémentaires paraît pertinente afin de parvenir à une compréhension globale du phénomène étudié.

\section{MÉTHODOLOGIE}

Le recours à une approche multicas se justifie par le caractère novateur des stratégies têtede-pont. Ce type d'approche permet d'accéder à un ensemble de données riches en détail, dont la précision serait difficilement atteignable par le biais de questionnaires. Elle s'avère particulièrement efficace pour analyser les événements dans leur contexte et renforce les interprétations du chercheur grâce à une plus grande diversité des données, permettant ainsi d'aller au-delà des spécificités des cas étudiés (Yin, 2003). Cette recherche est conduite sur la base d'entretiens semi-directifs d'une durée moyenne de 1 h 45 (de 40 minutes à 3 h 30)

3 Les IDE plateformes font référence aux stratégies combinant exportations et IDE. L'objectif de ce type d'investissement est de tirer profit des accords de libre-échange en implantant une filiale dans un pays de la zone destinée à desservir l'ensemble des marchés y appartenant. 
réalisés auprès de présidents-directeurs généraux et/ou directeurs de l’international de cinq PME manufacturières indépendantes de la région Rhône-Alpes détenant au moins une filiale à l'étranger, entre les mois de février et octobre 2012. Le choix de cette région se justifie par le poids du secteur industriel dans l'économie locale (deuxième région industrielle de France) ainsi que par le nombre important de PME manufacturières locales opérant à l'international. Face à la grande hétérogénéité existante en matière de PME, nous avons fait le choix de retenir la définition proposée par la Commission européenne en 2003, à savoir toute entreprise autonome réalisant un chiffre d'affaires annuel inférieur à 50 millions d'euros (ou un bénéfice net inférieur à 43 millions), composée d'un effectif inférieur à 250 salariés à temps plein. Les $\mathrm{PME}$ souffrant bien souvent d'un manque de ressources à consacrer à l'international, la concrétisation d'un IDE s'avère être une décision très lourde de conséquences - notamment dans le cas du secteur manufacturier - qu'il nous semble pertinent d'étudier. Précisons, à ce stade, que les entreprises manufacturières font l'objet d'un grand nombre de travaux dans la littérature en management international et leur étude a souvent abouti, dans des efforts de conceptualisation, au développement de cadres d'analyses, de modèles et autres concepts.

Réalisée avec le concours de l'organisme régional d'aide au développement international - ERAI (Entreprise Rhône-Alpes International) -, l'identification des PME de notre échantillon résulte d'une démarche en deux temps. Nous avons tout d'abord constitué une base de données répertoriant les 128 PME manufacturières indépendantes en Rhône-Alpes détenant au moins une filiale à l'étranger - via la consultation de bases de données financières telles que DIANE et d'archives de presse. Nous avons ensuite contacté puis interviewé les dirigeants des cinq PME retenues dans le cadre de notre échantillon - la constitution de celui-ci reposant sur une logique d'opportunisme méthodologique (Patton, 2002). Ces cas ont été spécifiquement sélectionnés sur la base de leur richesse en vue de maximiser le pouvoir contributif de notre recherche tout en conservant un maximum de flexibilité dans la collecte et le traitement des données.

Il a été demandé à chaque interviewé de répondre à un ensemble de questions relatives à l'identité de l'entreprise, à la décision de s'internationaliser, au choix de la localisation, au mode et à la vitesse d'internationalisation, à l'évaluation de la réussite (ou échec) de la stratégie mise en place, ainsi que les principaux projets de développement dans un horizon de trois ans. Chaque interview a fait l'objet d'un enregistrement puis d'une retranscription. Par souci de triangulation, les données récoltées ont ensuite été vérifiées en recourant à des sources secondaires telles que les sites Web des entreprises, les rapports financiers, des articles de presse, etc. Nous avons alors procédé à une analyse de contenu avec l'aide du logiciel N'Vivo. Cette phase nous a permis de mettre en avant les motivations au déploiement des stratégies tête-de-pont et, ainsi, donner du sens aux actions des acteurs interrogés. Le tableau 2 présente les principales caractéristiques des PME observées. 
TAbleau 2. CaractéristiQues De L'ÉCHANTILlon ${ }^{4}$

\begin{tabular}{|c|c|c|c|c|c|c|c|}
\hline PME & Activités & $\begin{array}{l}\text { Créa- } \\
\text { tion }\end{array}$ & $\begin{array}{l}\text { Effec- } \\
\text { tifs }\end{array}$ & $\begin{array}{l}\text { CA } \\
(\mathrm{M})\end{array}$ & $\begin{array}{l}\text { CA } \\
\text { export }\end{array}$ & $\begin{array}{l}1^{\text {re }} \\
\text { internat }\end{array}$ & $\begin{array}{l}\text { Principaux } \\
\text { marchés }\end{array}$ \\
\hline Claxtril & $\begin{array}{l}\text { Fabricant } \\
\text { d'équipements } \\
\text { extrusion } \\
\text { pour l'agro- } \\
\text { alimentaire, } \\
\text { la chimie et le } \\
\text { papier }\end{array}$ & 1956 & 210 & 42,5 & $73,8 \%$ & 1971 & $\begin{array}{l}87 \text { pays : } \\
\text { Europe, } \\
\text { Amérique } \\
\text { latine, Asie } \\
\text { du Sud-Est, } \\
\text { Australie, etc. }\end{array}$ \\
\hline Hydrol'o & $\begin{array}{l}\text { Fabricant } \\
\text { d'équipements } \\
\text { hydrauliques et } \\
\text { pneumatiques }\end{array}$ & 1971 & 20 & 3,7 & $40,5 \%$ & 2005 & $\begin{array}{l}60 \text { pays : } \\
\text { Afrique, } \\
\text { Europe, } \\
\text { Amérique } \\
\text { latine }\end{array}$ \\
\hline EGJ & $\begin{array}{l}\text { Fabricant } \\
\text { de joints } \\
\text { d'étanchéité }\end{array}$ & 1947 & n.d & 27,5 & $70,0 \%$ & 1990 & $\begin{array}{l}\text { Europe, } \\
\text { Océanie, } \\
\text { Amériques }\end{array}$ \\
\hline Macil & $\begin{array}{l}\text { Découpe } \\
\text { de matériel } \\
\text { électrique : } \\
\text { isolants souples, } \\
\text { câbles, gaines, } \\
\text { etc. }\end{array}$ & 1947 & 47 & 12,8 & $26,0 \%$ & 1975 & $\begin{array}{l}\text { PECO, Europe, } \\
\text { Moyen-Orient, } \\
\text { Afrique, Inde }\end{array}$ \\
\hline Flat & $\begin{array}{l}\text { Fabrication } \\
\text { d'appareillages } \\
\text { électriques liés } \\
\text { à la sécurité : } \\
\text { batteries, } \\
\text { onduleurs, } \\
\text { transformation } \\
\text { d'énergie }\end{array}$ & 1956 & 70 & 12,89 & $16,90 \%$ & 1969 & $\begin{array}{l}37 \text { marchés : } \\
\text { Allemagne, } \\
\text { Benelux, } \\
\text { PECO, } \\
\text { Scandinavie, } \\
\text { Afrique et } \\
\text { Moyen-Orient }\end{array}$ \\
\hline
\end{tabular}

\section{Claxtril}

Claxtril est une société spécialisée dans la fabrication de biens industriels pour les secteurs de l'agroalimentaire, de l'industrie papetière et chimique. Elle a été créée en 1956 suite à l'acquisition d'un brevet technique d'extrusion bivis rotative issu de l'industrie plastique. L'application de cette technologie au secteur agroalimentaire (notamment céréalier) en 1970 a permis à la PME de gagner un avantage concurrentiel notoire sur le marché français et

$4 \quad$ Les PME ayant été anonymisées sur demande de nos interlocuteurs, les noms présentés sont factices. 
de débuter son internationalisation par l'exportation à destination de l'Australie puis des États-Unis dès 1971 - soit un an après le virage stratégique pris par l'entreprise, faisant d'elle une born-again global selon les termes de Bell, McNaughton et Young (2001) et Bell et al. (2003).

Le choix des marchés cibles résulte d'un double constat : l'existence d'une demande pour la technologie et la similarité des habitudes de consommation des clients locaux et domestiques. La PME exporte désormais dans plus de 87 pays, dispose de deux filiales de production (Chili et Danemark) ainsi que d'une filiale commerciale en Inde, toutes trois contrôlées à $100 \%$. Les spécificités culturelles, la nature des matières premières disponibles, le protectionnisme local et la présence de certains clients historiques ont poussé la société à établir une unité de production au Chili, servant de tête-de-pont pour desservir les marchés sud-américains. Perçu comme étant le pays d'Amérique latine culturellement le plus proche du marché français, outre les différentes politiques locales d'attractivité des IDE, le Chili a rapidement retenu l'attention du président-directeur général. Le " made in Chile " permet, en effet, de contourner le protectionnisme douanier de marchés tels que le Brésil en bénéficiant des accords de coopération régionale, de gagner en compétitivité face aux producteurs locaux et d'augmenter ses marges. En d'autres termes, l'établissement d'une filiale tête-de-pont au Chili permet non seulement de se rapprocher d'une région commerciale à fort potentiel de croissance, mais aussi de réduire l'exposition aux risques institutionnels - de nature politique et d’affaires - et subjectifs liés à la méconnaissance des spécificités culturelles existantes sur le marché cible prioritaire (le Brésil).

"Dans la mesure du possible, nous passons par le Chili parce que si on peut donner de la valeur ajoutée à hauteur, au moins, de $40 \%$ du prix de vente à du matériel au Chili, on peut être estampillé "Made in Chile". Si vous êtes réputé "fabriqué au Chili", vous allez ensuite pouvoir profiter des accords Mercosur pour exporter vers le Brésil sans droits de douane. Par rapport au client final, un client brésilien peut gagner 20 à $30 \%$ du prix de vente s'il achète au Chili plutôt que d'acheter en France. » (président-directeur général de Claxtril)

L'existence de volumes d'affaires antérieurs récurrents et importants en Amérique latine s'est avérée être un élément déterminant dans la décision d'implantation dans la zone. Ceci atteste du rôle clé de l'expérience dans les choix stratégiques opérés en matière d'internationalisation. La mise en œuvre d'une stratégie tête-de-pont au Chili semble donc être davantage motivée par des préoccupations d'ordre économique et culturel, celle-ci permettant de desservir les marchés voisins en limitant les coûts et l'impact potentiellement négatif de la distance psychique. À l'inverse, l'ouverture de la filiale commerciale en Inde fait suite au retrait forcé de la PME du marché pakistanais, dû à la brusque dégradation de l'environnement local. Il s'agit donc, dans ce cas, d'une réponse stratégique à l'augmentation brutale et inattendue des niveaux de risque politique et social sur le marché cible. Le déploiement de la stratégie têtede-pont de l'Inde vers le Pakistan se justifie par les attentes de la PME en matière commerciale : anticipant une évolution positive à moyen terme, cette dernière tente de maintenir son positionnement au Pakistan en approvisionnant le marché par le biais de l'Inde.

"Le Pakistan, c'est un pays avec 170-180 millions d'habitants, donc on ne peut pas passer à côté du Pakistan. Ce qui est vrai aujourd'hui n'est pas forcément vrai demain [...] notre principal objectif, c'est d'être mieux implanté en Inde parce que c'est un marché très important, aux habitudes alimentaires assez anglo-saxonnes, ce qui correspond bien à nos produits. 
Aujourd'hui, il y a à peu près 150 millions d'habitants en Inde qui ont un niveau de vie ouesteuropéen, donc c'est très significatif. [...] Nous devons avoir une filiale en Inde pour développer les ventes dans tout le sous-continent indien parce qu'à partir de l'Inde, vous pouvez imaginer le Pakistan, le Bangladesh, etc. Ça fait beaucoup d'habitants. » (président-directeur général de Claxtril)

Soulignons que, pour des raisons historiques, l'Inde est culturellement plus proche de l'Europe que la grande majorité des pays de la zone et constitue un pont idéal entre les cultures occidentales et asiatiques, facilitant ainsi le développement de la PME dans le continent. L'ouverture de la filiale tête-de-pont peut être interprétée comme une stratégie réactive face aux risques institutionnels locaux. Elle permet de garantir l'accès aux marchés à forts potentiels tout en limitant l'exposition de la PME aux risques locaux.

\section{Hydrol'o}

Spécialisée dans la production et commercialisation d'équipements hydrauliques à destination des industries mécanique et métallurgique depuis 1971, Hydrol'o n’a débuté son internationalisation qu'en 2005. Malgré une conjoncture peu favorable, des produits matures et peu différenciés, elle enregistre une forte hausse des ventes à l'étranger ( 0 à $50 \%$ du chiffre d'affaires en provenance de l'export) entre 2005 et 2011 grâce à un recours accru aux TIC, renvoyant là encore au concept d'entreprise born-again global. La nature des pièces et des prestations de services complémentaires vendues par la société restreint ses possibilités d'expansion géographique par l'exportation.

"Il y a une part des activités que l'on peut exporter, mais c'est vrai que, contrairement au négoce, c'est très difficile d'aller proposer une partie de nos activités techniques au-delà d'un certain rayon. » (président-directeur général d'Hydrol'o)

La société a donc opté en 2008 pour la création d'une filiale tête-de-pont commerciale au Mexique afin de desservir les marchés d'Amérique du Sud ainsi qu'un bureau de représentation au Sénégal, à destination de l'Afrique de l'Ouest.

Le déploiement d'une stratégie tête-de-pont est motivé par le besoin d'Hydrol'o de se rapprocher des clients, d'accéder à deux régions émergentes à fort potentiel, bien que marquées par le poids des réseaux institutionnels et informels, en limitant les coûts de transaction. Précisons que les cas mexicain et sénégalais répondent à deux logiques différentes. Le déploiement de la stratégie tête-de-pont a été, dans le cas du Mexique, initié par un membre de l'entreprise disposant de la double nationalité franco-mexicaine. La maîtrise des subtilités linguistiques et la compréhension des spécificités culturelles présentent, en effet, l'avantage de réduire les effets négatifs de la distance psychique sur la PME. En cours d'industrialisation, le continent sud-américain paraît toutefois délaissé par les équipementiers et, donc, peu concurrentiel. Il s'agit ici d'une démarche proactive visant à permettre à Hydrol'o de bénéficier de l'avantage du premier entrant pour gagner d'importantes parts de marché et se positionner comme leader dans la zone. Inversement, la tête-de-pont commerciale sénégalaise est, pour sa part, justifiée par le manque de connaissances relatives au mode de fonctionnement des marchés d'Afrique de l'Ouest. Elle a donc pour principal objectif de réduire l'exposition de la PME aux risques d'origine subjective. 
Les efforts entrepris pour s'insérer dans les réseaux d'affaires locaux - prédominants dans la zone - permettent alors à la PME d'accumuler de l'expérience tout en bénéficiant de celle des membres du réseau, facilitant par là même l'identification de nouvelles opportunités. La confiance et l'expérience accumulées ont un impact notoire sur la stratégie d'expansion de l'entreprise, celle-ci envisageant d'ouvrir deux nouvelles filiales tête-de-pont (Tunisie et Chine) à destination des marchés émergents de l'Afrique du Nord et d'Asie du Sud-Est pour des raisons d'ordre culturel. Le président-directeur général explique que « c’est plus facile de travailler la Libye ou l'Égypte via la Tunisie qu'en direct, parce que la Tunisie a des relations plus simples avec ces pays-là que ne peut en avoir la France... C'est important d'arriver à comprendre ce maillage géopolitique, parce qu'en s'ouvrant des opportunités sur certains pays, ça nous ouvre aussi sur d'autres. D'où l'intérêt de s'implanter en Tunisie : ça reste quand même une porte ouverte non seulement sur le Maghreb, mais aussi sur tout le monde arabe et les pays du Golfe ».

\section{$E G J$}

EGJ est l'un des principaux fabricants européens de joints d'étanchéité à destination des industries cosmétiques, pharmaceutiques, agroalimentaires et chimiques. La faible différenciation des produits, entrés en phase de maturité, la taille restreinte du marché français et le statut de fournisseur exclusif de capsules métalliques auprès de certains clients situés à l'étranger sont les principaux éléments ayant conditionné le développement international de la PME. Quinze années d'exportations en Europe, en Amérique du Nord et du Sud puis en Océanie ont permis à l'entreprise de développer ses compétences et ses réseaux à l'étranger, attestant ainsi d'une internationalisation incrémentale comme décrite par l'école scandinave. Cette phase d'apprentissage s'avère nécessaire pour prendre conscience de l'importance de l'adaptation aux spécificités culturelles et contextuelles.

"La première phase est obligatoirement longue parce que vous devez véritablement bien vous imprégner de ce que représente la culture à l'étranger. Vous avez DES cultures : si vous n'êtes pas imprégné de ça et si vous précipitez l'implantation, vous allez vous planter. " (présidentdirecteur général d'EGJ)

L'ouverture d'une filiale de production en Chine en 2006 - tête-de-pont vers l'Océanie résulte du constat suivant : la réalisation de volumes d'affaires importants et récurrents dans la zone malgré un manque de compétitivité et réactivité face aux concurrents locaux, plus proches géographiquement, et produisant dans des pays à bas coûts de main-d'œuvre. La décision de localiser la production dans un pays émergent situé à mi-distance entre la France et l'Océanie permet à la PME de se rapprocher de ses clients les plus influents tout en bénéficiant d'une main-d'œuvre abondante à bas coût.

Comme l'indique le président-directeur général, la PME livrait "d'énormes volumes en Australie et Nouvelle-Zélande. [...] Un concurrent situé en Inde par exemple - à mi-chemin entre nous et [nos clients] - a quand même des avantages pour livrer plus facilement et à des prix plus compétitifs. En y réfléchissant, vous vous dites "si je veux pérenniser un chiffre d'affaires, il faut peut-être que je songe à créer un site secondaire un peu plus près de l'Australie". Soit vous le faites en Australie directement, soit vous vous dites qu'à mi-chemin, il y a des pays à bas coûts sur lesquels vous pourriez avoir un intérêt à produire localement pour, progressivement, en cas de coup dur, pouvoir vous retrancher et livrer de là-bas ". 
Le choix de la localisation de la filiale tête-de-pont repose sur trois facteurs cumulatifs, à savoir la stabilité environnementale, le potentiel de différentiation par rapport aux concurrents locaux et la possibilité d'exploiter un avantage technique conséquent.

"On a procédé un peu par élimination. En Inde, notre différenciation par rapport aux concurrents locaux était faible. On a regardé aussi la Chine, on a fait une petite étude de marché plus poussée. Je suis allé visiter les concurrents locaux et j'ai vu qu'on avait quand même un avantage technique encore important en Chine. C'est d'ailleurs ce qui a fait qu'on a pu lancer le site en Chine. Le Brésil n'était pas une option parce qu'on avait un concurrent fort local, et la Russie n'était pas une option parce qu'il y avait un risque fort en termes d'investissement, plus fort qu'en Chine en tous cas... Un risque mafieux, si vous voulez. " (président-directeur général d'EGJ)

Le dédoublement des sites de production est lié au besoin de diversifier les risques d'approvisionnement, bien que l'accès aux réseaux - institutionnels et d'affaires - et l'identification des membres clés demeurent deux préoccupations majeures de la PME. La sollicitation des relations actuelles permet en effet à la société de saisir un maximum d'opportunités ainsi que d'améliorer son positionnement ou de s'insérer dans de nouveaux réseaux locaux, légitimant par là le recours à une stratégie tête-de-pont pour desservir la zone pacifique. En d'autres termes, il s'agit ici d'une démarche proactive émanant du besoin de la PME de réduire son exposition aux risques d'affaires.

\section{Macil}

Macil est une entreprise spécialisée dans la transformation et distribution de matériaux isolants pour la construction électrique depuis 1947. Le caractère unique de ses innovations et savoir-faire, appréciés des multinationales clientes, a facilité son expansion géographique et lui assure aujourd'hui une position de leader sur son marché. La société s'est, en effet, rapidement orientée vers l'international afin de répondre aux sollicitations des clients étrangers. Le référencement en tant que distributeur exclusif auprès d'une FMN dans la zone Europe dans les années 70, puis Europe et Moyen-Orient (EMEA) dix ans plus tard, a entraîné une hausse considérable du volume d'affaires réalisé à l'étranger. Les mesures européennes de lutte contre les monopoles ayant poussé ce client à mettre fin à l'accord d'exclusivité, la PME s'est vue contrainte de reconsidérer sa stratégie en adoptant une démarche plus proactive à l'international.

Face au fort développement des pays d'Europe de l'Est et aux entrées massives de clients importants dans la zone, la PME a décidé d'établir une filiale de production en Pologne, têtede-pont à destination de la République tchèque, la Slovaquie, la Hongrie, les Balkans et la Russie. Le choix de la localisation dépend des caractéristiques du pays d'accueil et de l'image de l'entreprise en Europe centrale et orientale (PECOs), véhiculée par ses clients au sein de leurs propres réseaux. Fortement influencé par la nature des activités de transformation de matière première, le choix d'une localisation à proximité des clients s'avère nécessaire afin de gagner en réactivité et en compétitivité face aux producteurs locaux. La mise en œuvre de la stratégie tête-de-pont répond moins au besoin d'économiser les coûts de production que de rendre l'entreprise plus flexible. Le dédoublement des sites de production prévient ainsi le risque d'approvisionnement et permet, en outre, de répondre plus rapidement aux commandes des partenaires commerciaux. L'ancrage local vient alors pérenniser et développer 
les activités dans la zone PECO (pays d'Europe centrale et orientale) en optimisant l'allocation des ressources, à diversifier les risques opérationnels grâce à la création d'un second site de production et stockage, augmenter le pouvoir de négociation de la PME et améliorer sa capacité à contrôler son expansion géographique. En d'autres termes, le déploiement de la stratégie tête-de-pont relève d'une démarche réactive faisant suite à la perte d'un contrat d'exclusivité territoriale et lié au besoin de flexibilité de l'entreprise. Elle a pour principal objectif de réduire l'exposition aux risques d'affaires.

Outre la filiale d'Europe de l'Est, l'entreprise a récemment opté pour l'établissement d'un bureau commercial à Dubaï avec l'assistance d'ERAI, prémisse à la création d'une tête-depont commerciale au Moyen-Orient. Cette option vise à tester la réceptivité des marchés environnants en étant accompagnée dans ses choix par des experts français de la zone, bénéficier de la proximité géographique et des accords bilatéraux, tirer profit de l'absence constatée de concurrence locale et améliorer ses connaissances du pays en vue d'un développement plus engageant dans le futur. La position géographique stratégique de Dubaï, carrefour entre l'Europe, le Moyen-Orient et l'Asie, et les politiques locales d'attractivité territoriale (zones franches) s'avèrent être des éléments clés dans le choix de la localisation. Il est intéressant de noter que l'expérience réussie en Europe de l'Est semble avoir convaincu la PME de l'efficacité de la stratégie tête-de-pont sur les plans de la compétitivité et de la flexibilité. Si la première filiale tête-de-pont avait pour principale mission de réduire l'exposition aux risques d'affaires, il semble que l'expérience accumulée en Europe de l'Est a amené l'entreprise à modifier sa stratégie de pénétration des nouveaux marchés. En effet, l'implantation à Dubaï est moins liée à un besoin de réduire le niveau de risque supporté par la PME que d'adopter une stratégie nouvelle lui permettant de gagner un avantage concurrentiel solide au Moyen-Orient. Ainsi, les externalités positives découlant de la démarche défensive adoptée dans les PECOs ont permis d'institutionnaliser la stratégie tête-de-pont et d'en faire une stratégie proactive de pénétration de marché.

\section{Flat}

La société Flat est spécialisée dans la conception et distribution d'appareils électriques pour le secteur de la sécurité. CEuvrant sur un marché de niche lourdement impacté par le poids des normes techniques et disposant d'une solide réputation dans le milieu, la PME est le leader français dans son domaine. L'arrivée d'un nouveau dirigeant à la tête de l'entreprise au début des années 2000 a eu une incidence notoire sur la stratégie d'expansion de l'entreprise, passant d'une logique réactive à proactive en sélectionnant avec soin les marchés cibles et nouant des partenariats technologiques avec des multinationales étrangères. L'ouverture d'une filiale commerciale en Allemagne fin 2009 fait suite à la réalisation d'importants volumes d'affaires avec ses clients allemands, l'image positive du "made in Germany " et de l'expérience antérieure du dirigeant dans le pays.

Précédemment en poste en Allemagne, le dirigeant dispose d'un réseau personnel établi ainsi que d'une solide connaissance des spécificités culturelles locales, diminuant de fait le risque perçu de l'expansion dans la zone. La détention de certifications européennes, françaises et allemandes permet à la PME de se développer en Europe sans avoir à réaliser d'importants investissements - de temps et financiers - de mise en conformité. Il est intéressant de noter que si le choix du pays d'implantation apparait contraint par le champ d'application des normes et standards techniques, la sélection de la ville est influencée par la localisation 
d'ERAI dans le pays. L'entreprise a pu, dans les premières phases de son développement local, bénéficier du soutien de l'institution qui agit comme un catalyseur de l'expansion en ce qu'il limite l'exposition de la PME aux risques - notamment environnementaux et financiers.

La filiale allemande sert de tête-de-pont à destination des marchés culturellement proches, accordant davantage d'importance à la qualité technique et au respect des normes qu'au prix, dans lesquels certains de ses clients les plus importants sont solidement établis : l'Autriche et la Suisse allemande. L'entreprise souhaite bénéficier de la réputation et des réseaux de ses clients afin de pénétrer plus facilement et plus rapidement les marchés cibles, l'image de partenaire étant vecteur de crédibilité et de légitimité aux yeux des clients étrangers. Par ailleurs, l'ouverture progressive du marché polonais, son attrait pour les produits techniques certifiés TÜV ou VDS (normes allemandes) et l'identification d'un concurrent local direct ont également poussé la PME à considérer la filiale allemande comme tête-de-pont vers la Pologne.

"Ce côté induit du pays voisin (Allemagne-Pologne) peut nous aider à bâtir une plateforme de crédibilité : on peut, à mon avis, gagner 1 an par rapport à "la page blanche". La page blanche, c'est quand vous frappez à la porte, mais que personne ne vous connaît : vous n'avez pas de références, pas d'appui pour justifier votre arrivée. Une autre particularité de la Pologne, c'est que j'ai un concurrent direct en Pologne. C'est intéressant... C'est bien, c'est stimulant! Ça serait bien d'aller voir comment il se comporte "en vrai". Lui-même doit avoir des velléités sur d'autres pays, donc autant aller attaquer l'ennemi sur son terrain. Il connaît son terrain, je le reconnais, mais autant aller chez lui avant et le freiner chez lui avant qu'il ne soit arrivé chez moi. » (président-directeur général de Flat)

Eu égard aux spécificités du secteur d'activités, à la taille restreinte de la PME et au peu de ressources disponibles, la mise en œuvre de stratégie tête-de-pont s'avère pertinente afin d'optimiser l'allocation des ressources dédiées au développement international, mais s'effectue nécessairement par étapes.

"Je vais y aller étape par étape parce qu'il faut digérer la filiale. L'ouverture d'une filiale est liée quand même à la caractéristique du marché potentiel qu'on peut développer. Étant sur des marchés de niche, on ne peut pas partir n'importe où, le jeu n'en vaut pas la chandelle. Les objectifs suivants, c'est, à partir de la plateforme allemande, servir les pays de langue germanique c'est-à-dire l'Autriche et la Suisse allemande, pour des raisons stratégiques et aussi imposées par nos clients, parce qu'ils ont une forte propension à être présents en Autriche et en Suisse. [...] À partir de la plateforme allemande, je commence également à travailler mon prochain pays : la Pologne, avec ERAI. Pourquoi le choix de la Pologne? Par la taille géographique, le profil de développement du pays et une forme de conscience vis-à-vis de la norme. Il faut savoir que la Pologne est assez à cheval sur les normes et a d'ailleurs son propre organisme certificateur. » (président-directeur général de Flat)

Il s'agit ainsi d'une démarche proactive d'origine principalement interne, trouvant son fondement dans le besoin de différentiation de la PME et la volonté de consolider son réseau d’affaires. Le tableau 3 synthétise et complète les résultats précédemment présentés. 
TABleAU 3. SyNThèSE DES PRINCIPAUX RÉSUltats OBTENUS

\begin{tabular}{|c|c|c|c|c|c|c|c|}
\hline & $\begin{array}{l}\text { Modèle } \\
\text { d'internat }\end{array}$ & $\begin{array}{l}\text { Pays TDP } \\
\text { et type de } \\
\text { filiale }\end{array}$ & $\begin{array}{l}\text { Marché(s) } \\
\text { desservi(s) }\end{array}$ & $\begin{array}{l}\text { Nature } \\
\text { de la } \\
\text { démarche }\end{array}$ & $\begin{array}{l}\text { Risques/ } \\
\text { facteurs } \\
\text { exogènes }\end{array}$ & Facteurs endogènes & Motivations \\
\hline \multirow[t]{2}{*}{ Claxtril } & \multirow[t]{2}{*}{$\begin{array}{l}\text { Born } \\
\text { global }\end{array}$} & $\begin{array}{l}\text { Filiale de } \\
\text { production } \\
\text { contrôlée à } \\
100 \% \text { au } \\
\text { Chili }\end{array}$ & $\begin{array}{l}\text { Pays } \\
\text { membres du } \\
\text { Mercosur } \\
\text { (notamment } \\
\text { le Brésil) }\end{array}$ & Proactive & $\begin{array}{l}\text { Risques } \\
\text { d'affaires et } \\
\text { politiques : } \\
\text { protectionnisme, } \\
\text { spécificités } \\
\text { culturelles, } \\
\text { qualité et } \\
\text { disponibilité } \\
\text { des matières } \\
\text { premières }\end{array}$ & $\begin{array}{l}\text { - Détention } \\
\text { d'un avantage } \\
\text { technologique. } \\
\text { - Dépendance envers } \\
\text { les clients. } \\
\text { - Risques subjectifs : } \\
\text { méconnaissance des } \\
\text { spécificités locales. } \\
\text { - Volumes d'affaires } \\
\text { réalisés dans la } \\
\text { zone. }\end{array}$ & $\begin{array}{l}\text { - Améliorer la compétitivité face aux } \\
\text { concurrents locaux dans la zone : } \\
\text { - bénéficier des accords de } \\
\text { coopération régionaux ; } \\
\text { - contourner les barrières à l'entrée } \\
\text { (protectionnisme douanier). } \\
\text { - Proximité culturelle et accès } \\
\text { aux matières premières locales : } \\
\text { adaptation aux goûts des } \\
\text { consommateurs locaux et matières } \\
\text { premières disponibles localement. } \\
\text { - Suivi des entreprises leaders du } \\
\text { secteur dans la zone. }\end{array}$ \\
\hline & & $\begin{array}{l}\text { Filiale com- } \\
\text { merciale } \\
\text { contrôlée à } \\
100 \% \text { en } \\
\text { Inde }\end{array}$ & $\begin{array}{l}\text { Asie du Sud } \\
\text { (Pakistan et } \\
\text { Bangladesh) }\end{array}$ & Réactive & $\begin{array}{l}\text { Risques } \\
\text { institutionnels : } \\
\text { politiques } \\
\text { et sociaux } \\
\text { (tensions) }\end{array}$ & $\begin{array}{l}\text { Volumes d'affaires } \\
\text { existants dans la zone. }\end{array}$ & $\begin{array}{l}\text { - Garantir l'accès à des marchés à } \\
\text { forts potentiels, mais instables : } \\
\text { dégradation brutale de la stabilité } \\
\text { environnementale locale (guerre). } \\
\text { - Proximité culturelle et géographique } \\
\text { entre le pays tête-de-pont et la cible } \\
\text { + entre les pays d'origine et tête-de- } \\
\text { pont. }\end{array}$ \\
\hline
\end{tabular}




\begin{tabular}{|c|c|c|c|c|c|c|c|}
\hline & $\begin{array}{l}\text { Modèle } \\
\text { d'internat }\end{array}$ & $\begin{array}{l}\text { Pays TDP } \\
\text { et type de } \\
\text { filiale }\end{array}$ & $\begin{array}{l}\text { Marché(s) } \\
\text { desservi(s) }\end{array}$ & $\begin{array}{l}\text { Nature } \\
\text { de la } \\
\text { démarche }\end{array}$ & $\begin{array}{l}\text { Risques/ } \\
\text { facteurs } \\
\text { exogènes }\end{array}$ & Facteurs endogènes & Motivations \\
\hline \multirow[t]{2}{*}{ Hydrol'o } & \multirow[t]{2}{*}{$\begin{array}{l}\text { Born- } \\
\text { again } \\
\text { global }\end{array}$} & $\begin{array}{l}\text { Filiale com- } \\
\text { merciale } \\
\text { contrôlée à } \\
100 \% \text { au } \\
\text { Mexique }\end{array}$ & $\begin{array}{l}\text { Amérique } \\
\text { latine } \\
\text { (notamment } \\
\text { Pérou, } \\
\text { Chili) }\end{array}$ & Proactive & & $\begin{array}{l}\text { - Spécificité de } \\
\text { l'offre. } \\
\text { - Compétences des } \\
\text { ressources internes. } \\
\text { - Risques subjectifs : } \\
\text { pas d'expérience } \\
\text { directe dans la } \\
\text { région. }\end{array}$ & $\begin{array}{l}\text { - Diminuer les effets de la distance } \\
\text { psychique (langue, culture, } \\
\text { géographie). } \\
\text { - Opportunités internes : disposition } \\
\text { de RH qualifiée à double nationalité } \\
\text { (double culture). } \\
\text { - Améliorer la compétitivité face aux } \\
\text { concurrents locaux. } \\
\text { - Bénéficier des accords de } \\
\text { coopération régionaux. } \\
\text { - Recherche d'économie des coûts de } \\
\text { transaction : } \\
\text { - volumes d'affaires importants et } \\
\text { récurrents ; } \\
\text { - produits/offres difficilement } \\
\text { exportables au-delà d'un certain } \\
\text { périmètre. } \\
\text { - Mise à profit de l'expérience } \\
\text { antérieure accumulée. }\end{array}$ \\
\hline & & $\begin{array}{l}\text { Bureau } \\
\text { commercial } \\
\text { au Sénégal }\end{array}$ & $\begin{array}{l}\text { Afrique } \\
\text { de l'Ouest } \\
\text { (Côte } \\
\text { d'Ivoire, } \\
\text { Gabon) }\end{array}$ & Proactive & $\begin{array}{l}\text { Risques } \\
\text { d'affaires : } \\
\text { prédominance } \\
\text { des réseaux } \\
\text { d'affaires locaux }\end{array}$ & $\begin{array}{l}\text { Risques subjectifs : } \\
\text { pas de connaissance et } \\
\text { d'expérience dans la } \\
\text { région. }\end{array}$ & $\begin{array}{l}\text { Spécificités culturelles locales. } \\
\text { Connaissances spécifiques au marché } \\
+ \text { accès aux réseaux locaux. }\end{array}$ \\
\hline
\end{tabular}




\begin{tabular}{|c|c|c|c|c|c|c|c|}
\hline & $\begin{array}{l}\text { Modèle } \\
\text { d'internat }\end{array}$ & $\begin{array}{l}\text { Pays TDP } \\
\text { et type de } \\
\text { filiale }\end{array}$ & $\begin{array}{l}\text { Marché(s) } \\
\text { desservi(s) }\end{array}$ & $\begin{array}{l}\text { Nature } \\
\text { de la } \\
\text { démarche }\end{array}$ & $\begin{array}{l}\text { Risques/ } \\
\text { facteurs } \\
\text { exogènes }\end{array}$ & Facteurs endogènes & Motivations \\
\hline EGJ & Uppsala & $\begin{array}{l}\text { Filiale de } \\
\text { production } \\
\text { contrôlée à } \\
100 \% \text { en } \\
\text { Chine }\end{array}$ & Océanie & Proactive & $\begin{array}{l}\text { Risques } \\
\text { d'affaires }\end{array}$ & $\begin{array}{l}\text { - Caractéristiques } \\
\text { et maturité de } \\
\text { l'offre (faible } \\
\text { différentiation). } \\
\text { - Dépendance envers } \\
\text { les clients. } \\
\text { - Expérience } \\
\text { internationale. } \\
\text { - Volumes d'affaires } \\
\text { réalisés dans la } \\
\text { zone. }\end{array}$ & $\begin{array}{l}\text { - Améliorer la compétitivité face aux } \\
\text { concurrents locaux à bas coûts dans } \\
\text { la zone. } \\
\text { - Rapprochement des clients : } \\
\text { diminution coûts + délais livraison } \\
\text { donc gain de flexibilité + réactivité. } \\
\text { - Diversification des risques de } \\
\text { production : } 2 \text { sites = meilleure } \\
\text { absorption des chocs externes. } \\
\text { - Recherche d'économie des coûts de } \\
\text { transaction : } \\
\text { - volumes d'affaires importants et } \\
\text { récurrents ; } \\
\text { - produits faiblement différenciés et } \\
\text { en phase de maturité. } \\
\text { - Structure de la concurrence locale. } \\
\text { - Influence des réseaux : } \\
\text { - accès aux relations des membres } \\
\text { clés ; } \\
\text { - accès aux informations } \\
\text { spécifiques, pertinentes et fiables. } \\
\text { - Volonté de maîtriser le } \\
\text { développement international (rythme } \\
\text { et choix des marchés) : passage } \\
\text { d'une stratégie réactive à proactive. } \\
\text { - Vision stratégique : } \\
\text { - maximisation des profits ; } \\
\text { - réplication des stratégies réussies } \\
\text { à l'international. }\end{array}$ \\
\hline
\end{tabular}




\begin{tabular}{|c|c|c|c|c|c|c|c|}
\hline & $\begin{array}{l}\text { Modèle } \\
\text { d'internat }\end{array}$ & $\begin{array}{l}\text { Pays TDP } \\
\text { et type de } \\
\text { filiale }\end{array}$ & $\begin{array}{l}\text { Marché(s) } \\
\text { desservi(s) }\end{array}$ & $\begin{array}{l}\text { Nature } \\
\text { de la } \\
\text { démarche }\end{array}$ & $\begin{array}{l}\text { Risques/ } \\
\text { facteurs } \\
\text { exogènes }\end{array}$ & Facteurs endogènes & Motivations \\
\hline \multirow[t]{2}{*}{ Macil } & \multirow[t]{2}{*}{ Uppsala } & $\begin{array}{l}\text { Filiale de } \\
\text { production } \\
\text { contrôlée à } \\
100 \% \text { en } \\
\text { Pologne }\end{array}$ & $\begin{array}{l}\text { Pologne, } \\
\text { République } \\
\text { tchèque, } \\
\text { Slovaquie, } \\
\text { Hongrie, } \\
\text { Balkans et } \\
\text { Russie }\end{array}$ & Réactive & $\begin{array}{l}\text { Risques } \\
\text { d'affaires }\end{array}$ & $\begin{array}{l}\text { - Pérennité sur le } \\
\text { marché (perte d'un } \\
\text { contrat d'exclusivité } \\
\text { territoriale). } \\
\text { - Dépendance envers } \\
\text { les clients. } \\
\text { - Expérience } \\
\text { internationale. } \\
\text { - Volumes d'affaires } \\
\text { réalisés dans la } \\
\text { zone. }\end{array}$ & $\begin{array}{l}\text { - Suivi (et rapprochement) des clients. } \\
\text { - Diversification des risques } \\
\text { d'approvisionnement. } \\
\text { - Augmentation du pouvoir de } \\
\text { négociation. } \\
\text { - Gain de flexibilité et réactivité } \\
\text { (volonté d'ancrage local). } \\
\text { - Amélioration de la compétitivité de } \\
\text { l'entreprise. } \\
\text { - Contournement des risques locaux } \\
\text { (instabilité). } \\
\text { - Existence de volumes d'affaires } \\
\text { récurrents et importants dans la } \\
\text { zone. } \\
\text { - Taille et potentiel du pays/zone. } \\
\text { - Opportunité interne (RH spécialisée } \\
\text { ayant connaissance de la zone). }\end{array}$ \\
\hline & & $\begin{array}{l}\text { Bureau } \\
\text { commercial } \\
\text { à Dubaï } \\
\text { (future } \\
\text { filiale) }\end{array}$ & $\begin{array}{l}\text { Moyen- } \\
\text { Orient, Inde }\end{array}$ & Proactive & & $\begin{array}{l}\text { - Avantage du } \\
\text { premier entrant. } \\
\text { - Méconnaissance } \\
\text { des attentes du } \\
\text { marché cible. }\end{array}$ & $\begin{array}{l}\text { - Être le premier sur les marchés de } \\
\text { la zone } \\
\text { - Tester l.a réactivité du marché cible. } \\
\text { - Proximité géographique entre le } \\
\text { pays tête-de-pont et la cible : gain de } \\
\text { flexibilité. }\end{array}$ \\
\hline
\end{tabular}




\begin{tabular}{|c|c|c|c|c|c|c|c|}
\hline & $\begin{array}{l}\text { Modèle } \\
\text { d'internat }\end{array}$ & $\begin{array}{l}\text { Pays TDP } \\
\text { et type de } \\
\text { filiale }\end{array}$ & $\begin{array}{l}\text { Marché(s) } \\
\text { desservi(s) }\end{array}$ & $\begin{array}{l}\text { Nature } \\
\text { de la } \\
\text { démarche }\end{array}$ & $\begin{array}{l}\text { Risques/ } \\
\text { facteurs } \\
\text { exogènes }\end{array}$ & Facteurs endogènes & Motivations \\
\hline Flat & Uppsala & $\begin{array}{l}\text { Filiale com- } \\
\text { merciale } \\
\text { contrôlée à } \\
100 \% \text { en } \\
\text { Allemagne }\end{array}$ & $\begin{array}{l}\text { Pays ger- } \\
\text { maniques et } \\
\text { Pologne }\end{array}$ & Proactive & & $\begin{array}{l}\text { - Développement, } \\
\text { consolidation et } \\
\text { exploitation des } \\
\text { réseaux clients. } \\
\text { - Caractéristiques de } \\
\text { l'offre (besoin de } \\
\text { différentiation). } \\
\text { - Dépendance envers } \\
\text { les clients. }\end{array}$ & $\begin{array}{l}\text { - Caractéristiques spécifiques des } \\
\text { produits (normes.) } \\
\text { - Exigences spécifiques des clients } \\
\text { locaux. } \\
\text { - Diminution des effets de la } \\
\text { distance psychique (culture, langue, } \\
\text { géographie). } \\
\text { - Bénéficier de l'image positive du } \\
\text { pays TDP. } \\
\text { - Appui des réseaux institutionnels et } \\
\text { d'affaires. } \\
\text { - Gain d'avantage concurrentiel par la } \\
\text { mise à profit des connaissances de } \\
\text { l'international : } \\
\text { - connaissances expérientielles de } \\
\text { l'entreprise ; } \\
\text { - expérience internationale } \\
\text { antérieure + réseau du dirigeant }+ \\
\text { commerciaux locaux. } \\
\text { Volonté du dirigeant. }\end{array}$ \\
\hline
\end{tabular}




\section{RÉSULTATS ET DISCUSSION}

L'objectif de cet article est de montrer en quoi les stratégies tête-de-pont constituent un mode de gestion efficace des risques internationaux pour les PME. Dans un premier temps, il convient de souligner le caractère éclectique des stratégies tête-de-pont, celles-ci pouvant découler d'une démarche tant réactive que proactive et avoir trait à des activités de commercialisation comme de production. Qu'elles soient de nature défensive ou offensive, commerciale ou productive, il semble que le déploiement des stratégies tête-de-pont soit systématiquement conditionné par la préexistence de volumes d'affaires conséquents et récurrents dans la région cible. L'ensemble des dirigeants interviewés ont en effet attesté de l'existence d'importants volumes d'exportation dans la zone cible avant la création de leur filiale. Dès lors, il paraît pertinent d'affirmer que l'existence d'un volume d'affaires important constitue une condition préalable au déploiement des stratégies tête-de-pont.

Les entretiens réalisés dans le cadre de cette recherche nous ont amenés à constater que les stratégies tête-de-pont sont principalement déployées afin de se prémunir contre les risques de nature subjective et institutionnelle. Ces stratégies permettent aux PME de pallier les risques environnementaux inhérents aux mesures protectionnistes, à la qualité et à l'accessibilité des ressources et/ou des interlocuteurs locaux, aux spécificités culturelles, à l'instabilité locale ou encore au poids prépondérant des réseaux dans l'économie du marché cible. En lien avec Casson et Da Silva Lopes (2013), nos travaux mettent toutefois en avant le rôle prédominant des risques subjectifs, d'affaires, politiques et sociaux pour les PME. Celles-ci paraissent, en revanche, nettement moins impactées par les risques financiers et naturels. En d'autres termes, le recours aux stratégies tête-de-pont est justifié par l'exposition des PME aux risques subjectifs, d'affaires, politiques et sociaux, mais pas par les risques financiers et naturels. Nos travaux prolongent la classification proposée par Casson et Da Silva Lopes (2013) en y incluant la notion de réseau - dimension clé pour les PME au regard de leurs ressources limitées. La dimension réticulaire revêt aussi une importance particulière dans le cas des pays émergents dans la mesure où le fonctionnement de ces économies repose en majeure partie sur les réseaux informels (Meyer, Estrin, Bhaumik et Peng, 2009). Ces résultats confirment les propos de Johanson et Vahlne (2009) selon lesquels l'accès au réseau d'affaires agit comme un facilitateur de l'internationalisation des entreprises et que la nonappartenance au réseau constitue un frein majeur.

Par ailleurs, il ressort de notre recherche que les stratégies tête-de-pont constituent à la fois un mode de gestion des risques et un vecteur de nouvelles opportunités d'affaires à l'international. Elles permettent, tout d'abord, aux PME ayant une démarche défensive de se prémunir contre tout élément externe pouvant nuire à leur compétitivité (voire à leur pérennité) sur les marchés cibles. Les stratégies tête-de-pont ont ici pour mission de limiter l'exposition des entreprises aux facteurs exogènes (ou "risques négatifs ») pour lesquels l'évolution est difficilement prévisible et/ou contrôlable. Inversement, ces stratégies permettent aux entreprises suivant une logique offensive d'être proactives à l'international et de saisir de nouvelles opportunités d'affaires à l'étranger en se positionnant - en amont - sur des marchés à fort potentiel. Elles apparaissent alors essentiellement motivées par des éléments propres à l'entreprise tels que les caractéristiques de l'offre et son degré de maturité, l'expérience internationale antérieure de l'entreprise et/ou de ses membres, les relations du réseau ou encore la vision du dirigeant. Les stratégies tête-de-pont font, dans ce cas, office de catalyseur de 
l'expansion internationale, la prise de risques «positifs » favorisant l'accès à de nouvelles opportunités, donc la diversification du portefeuille marché des PME.

Le tableau 4 résume les risques « négatifs » et « positifs » mentionnés par les cinq PME et auxquels les stratégies tête-de-pont ont permis d’apporter une réponse efficace.

TAbleau 4. Synthèse DeS RISQUeS AUXQUels RÉPONDENT LeS STRATÉGIeS TÊTE-DE-PONT

\begin{tabular}{ll}
\hline Nature du risque & Composants \\
\hline Risques «négatifs » & Disponibilité des ressources humaines et physiques \\
& Sécurité de l'approvisionnement \\
& Instabilité politique et sociale \\
& Protectionnisme douanier \\
& « Handicap de la firme étrangère » au pays et aux réseaux \\
\hline Risques «positifs» & Exploitation des avantages techniques et/ou prolongation du cycle de vie \\
& des produits \\
& Exploitation et consolidation des réseaux \\
& Avantage du premier entrant dans la zone \\
& Externalités positives liées à la proximité culturelle (pays tête-de-pont/ \\
& pays cible) \\
& Diversification des risques d'affaires (clients, fournisseurs, \\
& approvisionnement) \\
& Différentiation et valorisation de l'entreprise (réputation du pays tête-de- \\
& pont, etc.) \\
&
\end{tabular}

Ce tableau s'inscrit dans la lignée des travaux de Figueira-de-Lemos, Johanson et Vahlne (2011) quant à la prise de risque volontaire des entreprises dans une logique de maximisation de la valeur. Le cas de EGJ montre, par exemple, bien qu'averses aux risques, certaines PME s'exposent volontairement à un certain niveau de risques qu'elles estiment prévisibles, supportables et maîtrisables afin de maximiser leurs profits. Il est toutefois à noter qu'EGJ limite son exposition en implantant sur place des technologies matures, non stratégiques pour l'entreprise, et conserve les activités génératrices de valeur ajoutée sur son marché d’origine.

Le cas d'EGJ montre que les stratégies tête-de-pont facilitent l'accès des PME à de nouveaux marchés - notamment émergents, à fort potentiel et encore peu concurrentiels tout en limitant leur exposition aux risques locaux. En d'autres termes, la stratégie tête-de-pont constitue à la fois une protection contre les risques et un vecteur d'opportunités d'affaires.

\section{CONCLUSION}

L'internationalisation des PME connaît un regain d'intérêt dans la littérature en management international. Souvent décrites comme subissant les pressions de l'environnement et manquant de ressources, elles tendent désormais à adopter des stratégies semblables à celles employées par les grands groupes, n'hésitant pas à s'engager de façon conséquente sur les marchés étrangers. Les entreprises se développant à l'international tendent traditionnellement à 
suivre un processus d'engagement graduel sur les marchés face au manque de connaissances expérientielles et au besoin de minimisation de l'incertitude. Si la distance psychique était le pivot de l'approche processuelle défendue par l'école scandinave, le modèle d'Uppsala revisité insiste désormais sur l'insertion dans les réseaux d'affaires comme principal moteur de l'internationalisation (Johanson et Vahlne, 1977, 2009 ; Vahlne et Johanson, 2013). Cette vision s'oppose néanmoins à celle des entreprises born-global selon laquelle la maximisation de la valeur prime sur la minimisation de l'incertitude.

L’optimisation nécessaire des ressources et les conditions environnementales locales obligent les PME à revoir leurs stratégies de localisation. Le choix de la localisation ne repose pas simplement sur les caractéristiques spécifiques du marché, mais semble bien influencé par la capacité du pays à servir de porte d'accès vers d'autres marchés. Ceci oblige l'entreprise à procéder à une analyse plus large et implique qu'elle ait accès à un ensemble d'informations pertinentes guidant la prise de décision. La méconnaissance des spécificités locales, le manque de transparence de l'environnement et l'aversion au risque sont autant de contraintes que les PME parviennent à contourner en mobilisant les réseaux institutionnels et d'affaires. L'adoption d'une stratégie tête-de-pont constitue une alternative pertinente permettant aux PME de poursuivre leur expansion internationale tout en limitant l'exposition aux risques locaux et/ou l'engagement de ressources supplémentaires. Cela suppose toutefois l'existence préalable de transactions importantes et récurrentes sur le long terme avec le pays cible. Ce type d'approche s'avère particulièrement efficace en contexte protectionniste (notamment douanier et normatif) ou d'instabilité environnementale. Le déploiement des stratégies tête-de-pont s'explique, dans un premier temps, par la volonté des PME de tirer avantage des accords internationaux et de l'image du pays d'implantation ainsi que d'éviter la concurrence frontale avec les acteurs locaux. Elle trouve également son fondement dans la nature des produits et leur degré de maturité, les connaissances expérientielles développées, les relations des réseaux ou encore la vision du dirigeant. Il ressort de cette étude que la stratégie tête-de-pont constitue une alternative viable à la réalisation de nouveaux IDE dans les marchés périphériques, répondant ainsi à la problématique antagoniste de l'accès aux marchés étrangers et de la minimisation des risques.

Par leur caractère novateur, les stratégies tête-de-pont demeurent relativement peu étudiées dans la littérature en management international. Comme le précisent Javalgi et al. (2010, p. 210), «bien que différents facteurs clés influençant les décisions relatives aux modes d'entrées aient été identifiés dans la littérature, la question de l'investissement dans un pays servant de tête-de-pont à d'autres économies émergentes n'a jamais été étudiée ». Notre recherche apporte un premier éclairage quant aux effets du déploiement des stratégies tête-de-pont sur la réduction des risques supportés par les PME qui s'internationalisent. Il conviendrait de répliquer cette analyse sur un échantillon plus large, de procéder à une segmentation du secteur manufacturier afin d'observer l'impact de l'intensité capitalistique et/ou technologique de celui-ci sur l'adoption de la stratégie et le choix de la localisation du pays tête-de-pont. De surcroît, la recherche ayant été menée dans le cadre des PME de la région Rhône-Alpes, il serait opportun d'étendre nos travaux aux entreprises d'autres régions et pays. Cela permettrait de mettre en exergue le rôle des organismes régionaux d'appui au développement international ainsi que d'identifier d'éventuelles similarités ou différences dans le choix de la localisation. Enfin, il serait intéressant de mener une analyse comparative à grande échelle afin de mettre en lumière quelques éléments clés nous permettant d'établir une méthode d'identification et de classification des pays tête-de-pont. 


\section{RÉFÉRENCES}

Andersen, O. (1993). On the internationalization process of firms : a critical analysis. Journal of International Business Studies, 24(2), 209-231.

Angué, K. et Mayrhofer, U. (2010). Le modèle d'Uppsala remis en question : une analyse des accords de coopération noués dans les marchés émergents. Management International, 15(1), 33-46.

Bell, J., McNaughton, R. et Young, S. (2001). « Born-again global » firms - An extension to the «born global » phenomenon. Journal of International Management, 7(3), 173-189.

Bell, J., McNaughton, R., Young, S. et Crick, D. (2003). Towards an integrative model of small firm internationalisation. Journal of International Entrepreneurship, 1(4), 339-362.

Bertrand, J. et St-Pierre, J. (2015). L'influence des caractéristiques des PME sur leur capacité à identifier les risques dans leurs projets de développement de produits. Colloque Risque et PME. Lyon, France, $1^{\text {er }}$ et 2 avril.

Casson, M. et Da Silva Lopes, T. (2013). Foreign direct investment in high-risk environments : an historical perspective. Business History, 55(3), 375-404.

Coviello, N.E. et Munro, H.J. (1997). Network relationships and the internationalization process of small software firms. International Business Review, 6(4), 361-386.

Coviello, N.E. et McAulley, A. (1999). Internationalization and the small firm : a review of contemporary empirical research. Management International Review, 39(3), 223-237.

Cyert, R.D. et March, J.G. (1963). A behavioral theory of the firm. Englewood Cliffs, New Jersey, Prentice Hall.

Ekholm, K., Forslid, R. et MARkusen, J.-R. (2007). Export-platform foreign direct investment. Journal of the European Economic Association, 5(4), 776-795.

Evans, J. et Mavondo, F.T. (2002). Psychic distance and organizational performance : an empirical examination of international retailing operations. Journal of International Business Studies, 33(3), 515-532.

Figueira-De-Lemos, F., Johanson, J. et VAhlne, J.-E. (2011). Risk management in the internationalization process of the firm : a note on the Uppsala model. Journal of World Business, 46, 143-153.

ForsGren, M. (2002). The concept of learning in the Uppsala internationalization process model : a critical review. International Business Review, 11(3), 257-277.

Gabrielsson, M., Kirpalani, V.M., Dimitratos, P., Solberg, C.A. et Zucchella, A. (2008). Born globals : propositions to help advance the theory. International Business Review, 17(4), 385-401.

INSEE (2015). Mondialisation, compétitivité et innovation - Les entreprises en France. Insee Références.

Javalgi R.G., Deligonul, S., Ghosh, A.K., Lambert, D.M. et Cavusgil, S.T. (2010). Foreign market entry mode behavior as a gateway to further entries : the NAFTA experience. International Business Review, 19(1), 209-222.

Jean-Amans, C. (2011). Contrôle et coordination des filiales des PME françaises à l'étranger (thèse de doctorat en sciences de gestion). Université Toulouse 1 Capitole.

Johanson, J. et Vahlne, J.-E. (1977). The internationalisation process of the firm - A model of knowledge development and increasing foreign market commitments. Journal of International Business Studies, 8(1), 23-32.

JoHANSON, J. et VAHLNE, J.-E. (2009). The Uppsala internationalization process model revisited : from liability of foreignness to liability of outsidership. Journal of International Business Studies, 40(9), 1411-1431. 
Johanson, J. et Wiedersheim-Paul, F. (1975). The internationalization of the firm - Four swedish cases. Journal of Management Studies, 12(3), 305-323.

Lemaire, J.-P. (2013). Stratégies d'internationalisation : nouveaux enjeux d'ouverture des organisations, des activités et des territoires ( $3^{e}$ édition). Paris, Dunod.

Leyronas, C. et Torrès, O. (1996). Stratégie de mondialisation et PME : l'instruction d'un paradoxe. $3^{e}$ Congrès international francophone de la PME. Trois-Rivières, Québec.

Lu, J.W. et BeAmish, P.W. (2001). The internationalization and performance of SMEs. Strategic Management Journal, 22(6-7), 565-586.

Meier, O. et Meschi, P.X. (2010). Approche intégrée ou partielle de l'internationalisation des firmes : les modèles Uppsala (1977 et 2009) face à l'approche « international new venture » et aux théories de la firme. Management International, 15(1), 11-18.

MÉTAis, E., Véry, P. et Hourquet, P.-G. (2010). Le paradigme d’Uppsala : la distance géographique et l'effet de réseau comme déterminants des décisions d'acquisitions internationales (1990-2009). Management International, 15(1), 47-58.

Meyer, K.E., Estrin, S., Bhaumik, S.K. et Peng, M.W. (2009). Institutions, resources, and entry strategies in emerging economies. Strategic management journal, 30(1), 61-80.

Minda, A. et Nguyen, H.-T.-T. (2012). Les déterminants de l'investissement direct étranger d'exportation-plateforme. Revue économique, 63, 69-91.

NAнApiet, J. et GHoshal, S. (1998). Social capital, intellectual capital and the organizational advantage. Academy of Management Review, 23(2), 242-267.

Nguyen, H.-T.-T. (2011). L'investissement direct étranger d'exportation-plateforme : des facteurs de localisation aux impacts sur l'économie d'accueil : le cas des industries manufacturières au Vietnam (thèse de doctorat en sciences économiques). Université Toulouse 1 Capitole.

OJala, A. et TyrväInen, P. (2007). Market entry and priority of small and medium-sized enterprises in the software industry : an empirical analysis of cultural distance, geographic distance, and market size. Journal of International Marketing, 15(3), 123-149.

Oviatt, B.M. et McDougall, P.P. (1994). Toward a theory of international new ventures. Journal of International Business Studies, 25(1), 45-64.

Pantin, F. (2010). Conduire l'internationalisation des PME : un processus porté par les compétences de l'équipe dirigeante. Revue internationale PME, 23(3-4), 13-42.

Patton, M.Q. (2002). Qualitative research and evaluation methods. Thousand Oaks, Sage Publications.

St-Pierre, J., Defays, L., Benezech, D. et Garcia, C. (2014). Le rôle du capital humain dans l'établissement de collaborations internationales des PME. Management International, 18, 72-84.

VAhlne, J.-E. et Johanson, J. (2013). The Uppsala model on evolution of the multinational business enterprise-from internalization to coordination of networks. International Marketing Review, 30(3), 189-210.

Weerawardena, J., Mort, G.S., Liesch, P.W. et Knight, G. (2007). Conceptualizing accelerated internationalization in the born global firm : a dynamic capabilities perspective. Journal of World Business, 42(3), 294-306.

YIN, R. (2003). Case study research. Design and methods (3édition). Beverly Hills, Sage Publications. 